\title{
N-Heterocyclic Carbene/Magnesium Co-catalyzed Radical Relay Assembly of Aliphatic Keto-nitriles
}

\author{
Lei Chen," Shiyi Jin," Jian Gao, Tongtong Liu, Yuebo Shao, Jie Feng, ${ }^{*}$ Kangyi Wang, \\ Tao Lu, Ding Du*
}

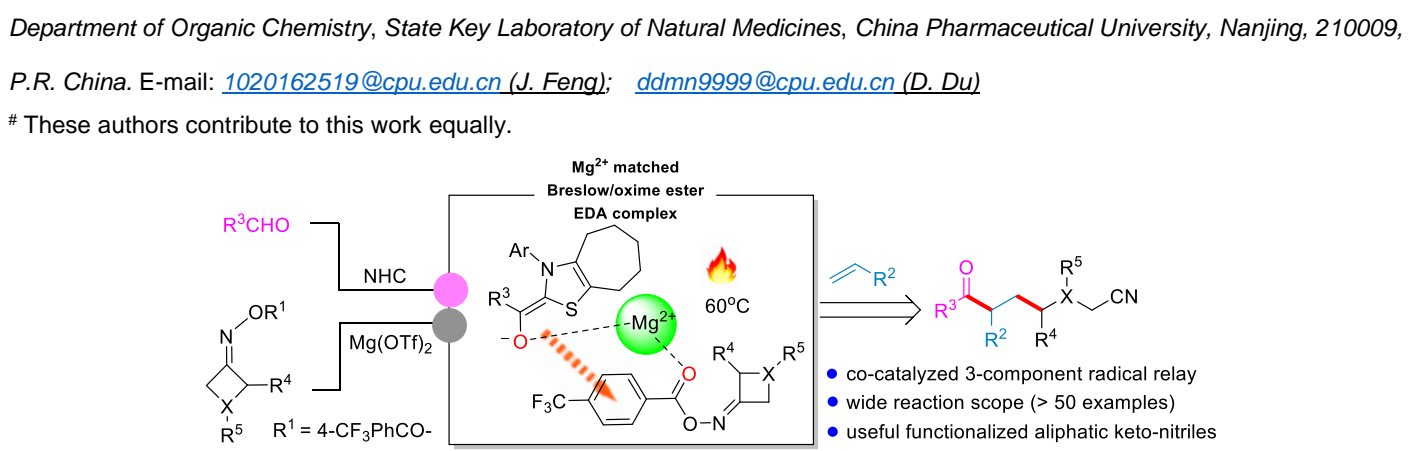

Abstract: An N-heterocyclic carbene and magnesium co-catalyzed three-component alkylacylation of alkenes with cycloketone oxime esters and aldehydes was presented. This method displayed good scope generality, providing a transition metal and photo-redox free pathway to access various multi-functionalized aliphatic keto-nitrile structures under mild reaction conditions. Moreover, this strategy is supposed to follow a radical relay mechanism via a single electron transfer (SET) event of Mg/oxime ester/Breslow intermediate ternary electron donating acceptor (EDA) complex.

Aliphatic compounds bearing multi-functional groups represent key structures in many chemical frontier fields. For example, they are the basic skeletons for dual target inhibitors, ${ }^{1}$ the chemical linkers for surfactants or polymers, ${ }^{2}$ and the connectors for supported catalysts ${ }^{3}$ (Scheme 1a). Traditional synthesis of such compounds usually required the installation of each functional group in sequence, which was confronted with extra workloads such as protection/deprotection procedures, selectivity issues and work-up problems.

Multicomponent reactions (MCRs) have been recognized as a powerful tool to provide a straightforward access to various complex structures. ${ }^{4}$ Among them, the intermolecular dicarbofunctionalization of olefins, installing a sp ${ }^{3}$-alkyl group and another carbon group into the substrates, represents one of the most efficient MCR strategies to enable the streamlined construction of aliphatic frameworks in a single step. ${ }^{5}$ Regularly, transition metal (TM) catalysis, ${ }^{6}$ especially $\mathrm{Pd}^{7}$ and more recently $\mathrm{Ni}$ catalysis, ${ }^{8}$ have been successfully applied to these transformations (Scheme 1b). However, the in situ generated alkyl metal intermediates may undergo 
potential side reactions such as $\beta-\mathrm{H}$ elimination and isomerization. Despite the significant progress in TMcatalyzed reactions, catalytic transition-metal free dicarbofunctionalization especially alkylacylation of alkenes remains highly desired.

$\mathrm{N}$-Heterocyclic carbenes (NHCs) have emerged as one of the leading organocatalysts, especially for the characteristic ability to access umpolung reactivity of diverse aldehydes. ${ }^{9}$ In recent years, NHC-catalyzed reactions via a radical process have evoked great interest among organic chemists, ${ }^{10}$ which led to the development of few NHC-catalyzed radical relay reactions for olefin dicarbofunctionalization (Scheme 1b). ${ }^{11}$ In 2019, the Ohmiya group applied the NHC-catalyzed radical relay into a three-component coupling of aldehydes, alkenes and tertiary alkyl carboxylic acid-derived redox-active esters. ${ }^{11 a}$ They lately reported the vicinal dicarbofunctionalization of alkenes using aldehydes and tertiary $\alpha$-bromo esters. ${ }^{11 b}$ In 2020 , The Hong group demonstrated a deaminative process using redox active Katritzky pyridinium salts, in which some dicarbofunctionalization examples were described. ${ }^{11 c}$ During this period, NHC-catalyzed three-component acylfluoroalkylation of alkenes has also been reported. ${ }^{11 \mathrm{~d}-\mathrm{f}}$ Although these NHC-catalyzed radical relay processes have enabled the elegant TM-free organic transformations via electron donating acceptor (EDA) strategy, it is still in high demand to further discover novel matched substrate pairs and reaction modes. We noticed that cycloketone oxime esters are useful and versatile intermediates in organic synthesis, which serve as the feedstock for the synthesis of functionalized nitriles via the formation of nitrile alkyl radicals under TM catalysis or photoredox conditions. ${ }^{12}$ We conceived that the cyclo ketone oxime esters incorporation with alkenes and aldehydes under NHC catalysis might in principle afford the target aliphatic keto-nitriles via the EDA strategy (Scheme 1c). In particular, the newly formed nitrile group can allow various further transformations that would be useful in synthetic and medicinal chemistry. However, it was found that the reductive potential of cyclo ketone oxime esters were recorded around $-1.6 \mathrm{~V}$ (VS SCE in $\mathrm{MeCN}),{ }^{12 \mathrm{~d}}$ while the reductive potential of enolate (deprotonation of the Breslow intermediate) was around -0.95 $\sim-0.97 \mathrm{~V}$ (vs SCE in MeCN). ${ }^{13}$ It seems that the enolate might not attain sufficient reduction potential for the cyclic oxime esters. Since NHC/Lewis acid (LA) co-catalysis has been widely used to enable numerous unconventional transformations, we assumed that the electron transfer of this theoretically mismatched species might occur with the assistance of a Lewis acid. Herein, we wish to present a magnesium promoted NHCcatalyzed radical relay protocol to patch up the potential gap between cycloketone oxime esters, aldehydes and alkenes, providing a facile access to functionalized aliphatic keto-nitriles (Scheme 1c). 


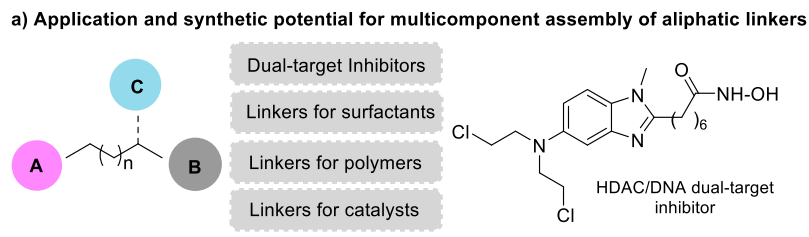

b) $\mathrm{C}\left(\mathrm{sp}^{3}\right)-\mathrm{C}$ olefin dicarbofunctionalization

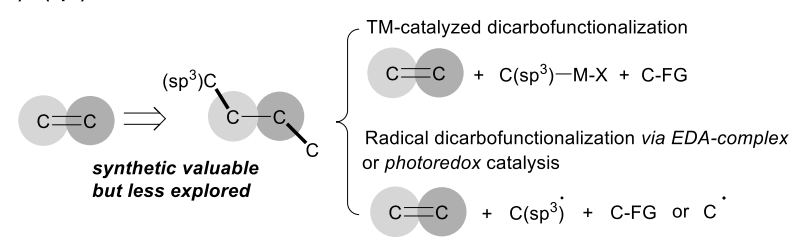

c) This work: multicomponent assembly of aliphatic linkers via Mg/Oxime ester/Breslow intermediate EDA complex

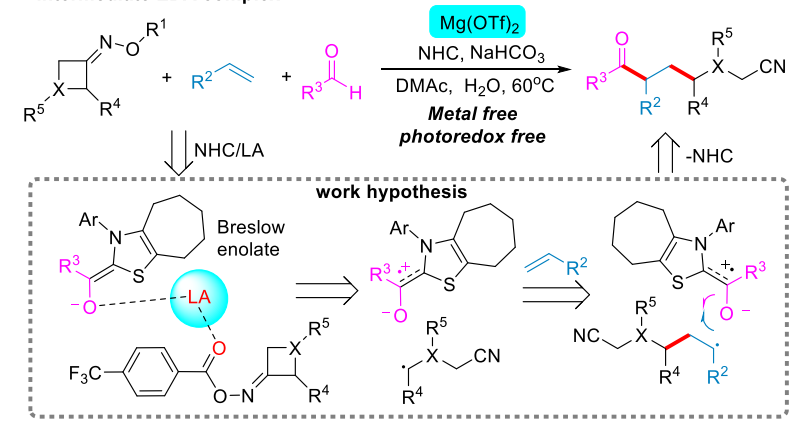

Scheme 1. Overview for dicarbofunctionalization of olefins and our work hypothesis.

To start this work, cyclobutanone oxime ester $\mathbf{1 a}$, styrene $\mathbf{2 a}$ and 4 -chlorobenzaldehyde $\mathbf{3 a}$ were used as the model substrates (Figure 1). The standard condition was identified as the one resulting in $71 \%$ isolated yield after the screening of different experimental parameters (SI, Table S1-S5). No product was observed in the absence of an $\mathrm{NHC}$ catalyst (Figure 1, line 1). The presence of $\mathrm{Mg}(\mathrm{OTf})_{2}$ was also crucial to this $\mathrm{MCR}$ reaction with otherwise a low yield observed (Figure 1, line 2). La(OTf) $)_{3}$ could slightly enhance the reaction yield to $23 \%$ (Figure 1, line 3), while other tested Lewis acids led to no acceleration (SI, Table S3). To our interest, the water content (2.0 equiv) was found to be another key factor for the success of this reaction because using super dry solvent along with molecule sieves or adding more water (i.e. 20 equiv) always gave inferior results (Figure 1, line 4-5). Water may act as the activator for oxime esters, ${ }^{14}$ and meanwhile $\mathrm{Mg}(\mathrm{OTf})_{2}$ is prone to absorb moisture to form hydrated magnesium salt, which may affect the reactivity of magnesium ions in organic solvent. Further screening of the bases identified the weak base $\mathrm{NaHCO}_{3}$ as the optimal one, and poor conversions were observed for both strong organic and inorganic bases (Figure 1, line 6-7). Additionally, conducting the reaction under air or at room temperature was found to be ineffective (Figure 1, line 8-9). 

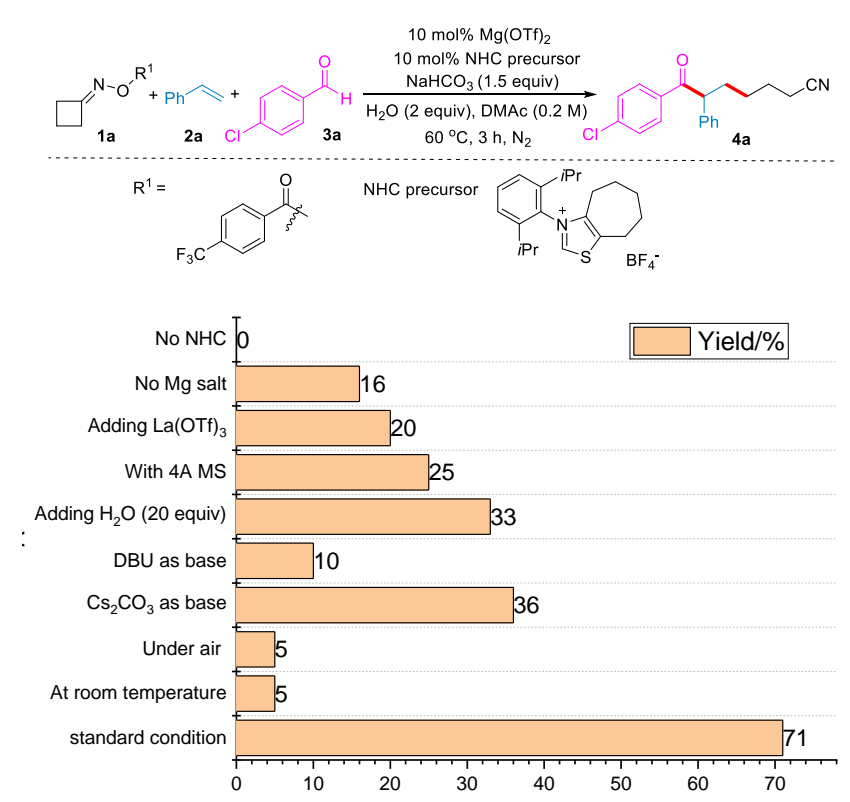

Figure 1. Condition modifications for NHC-catalyzed radical relay alkylacylation of alkenes. Standard conditions: cyclobutanone oxime ester $\mathbf{1 a}(0.45 \mathrm{mmol})$, styrene $\mathbf{2 a}(1.5 \mathrm{mmol})$ and benzaldehyde $3 \mathbf{a}(0.3 \mathrm{mmol}), \mathrm{NHC}$ precursor $(0.03 \mathrm{mmol}), \mathrm{NaHCO}_{3}(0.45 \mathrm{mmol}), \mathrm{H}_{2} \mathrm{O}$ (0.6 mmol), dry DMAc $(3 \mathrm{~mL}), 60^{\circ} \mathrm{C}, 4 \mathrm{~h}$. Isolated yields are based on $\mathbf{3 a}$.

With the optimized conditions in hand, the substrate scope was then investigated. The reactions of diverse olefins 2 with oxime ester $\mathbf{1 a}$ and 4-chlorobenzaldehyde $\mathbf{3 a}$ were initially tested (Scheme 2). Excess olefins were usually required to reach the best yields owing to their high volatility. Overall, substituted styrenes showed good tolerance with the isolated yield range from $54 \%$ to $85 \%$ (4b-40). Particularly, the reactions of sterically hindered 2-substituted styrenes and 1-vinylnaphthalene proceeded smoothly to give the corresponding products $\mathbf{4 n - 4 p}$ in moderate yields. The reaction of 4-vinylpyridine was also found to be feasible although with a slightly decreased yield (4q). Notably, the intermolecular olefin alkylacylation was seldom applied to activated olefins such as acrylates because the side Michael addition reactions were prone to occur. Gratifyingly, the desired products $\mathbf{4 r - 4 t}$ were obtained in moderate yields for several acrylates. However, phenyl acrylate was not suitable to this protocol $(\mathbf{4 u})$. It was noteworthy that the acrylate bearing a perfluorocarbon chain was also applicable to this method although with a lower yield. The obtained product $\mathbf{4 v}$ might have potential application in fluorine chemistry..$^{15}$ To further demonstrate the utility of this protocol, some pre-synthesized olefins bearing natural or bioactive fragments such as cholesterol and estrone were then screened. To our delight, they produced the desired products $4 \mathbf{w}$ and $\mathbf{4 x}$, respectively in $28 \%$ and $60 \%$ yield. 


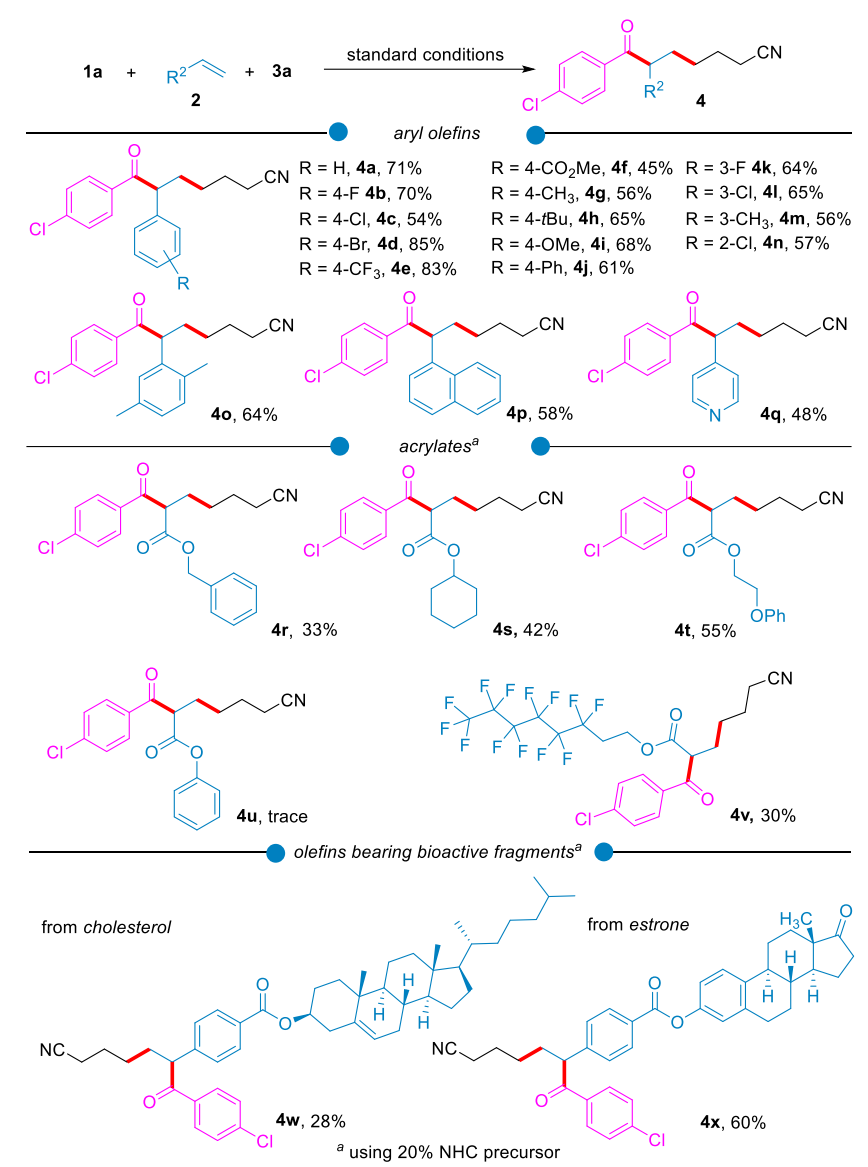

Scheme 2. Substrate scope for diverse aryl olefins and acrylates.

Subsequently, the generality of aryl aldehydes was investigated (Scheme 3). The substitents of the aryl aldehydes were found to have great impact on the reaction yields. The yields varied widely from $41 \%$ to $73 \%$ (5a-5n), perhaps owing to the varied reactivity and redox potential of the Breslow intermediates generated via the combination of aryl aldehydes with the NHC. Overall, some of the para-substituted benzaldehydes gave superior yields than that bearing meta-substituents (5a-5c and $\mathbf{5 f}$ vs $\mathbf{5 j - 5 n})$. For some cases, increase of the $\mathrm{NHC}$ pre-catalyst loading could slightly enhance the reaction yields ( $\mathbf{5 d}$ and $\mathbf{5 h} \mathbf{\mathbf { 5 }} \mathbf{\mathrm { I }})$. The more sterically hindered 2-Me benzaldehyde and 1-naphthaldehyde were inapplicable to this protocol (5o and $\mathbf{5 p}$ ), while the less sterically hindered 2-naphthaldehyde was tolerated to afford product $\mathbf{5 q}$ in $44 \%$ yield. Then, several representative heterocyclic aromatic aldehydes were examined. The reaction of 2-furyl and 2-thienyl aldehydes proceeded smoothly to afford the desired products $5 \mathrm{r}$ and $\mathbf{5 s}$, both in $59 \%$ yield. Decreased yields were obtained with nicotinaldehyde, benzo[b]thiophene-2-carbaldehyde and benzofuran-2-carbaldehyde $(\mathbf{5 t - 5 v})$ due to the poor conversion of the aldehyde substrates. However, in terms of quinoline-2-carbaldehyde, the undesired compound $\mathbf{5}$ ' derived from the two-component coupling of the oxime ester with the aldehyde was obtained as the major product. Moreover, aldehydes bearing bioactive fragments were found to be suitable for this 
transformation. Products $\mathbf{5 x}$ and $\mathbf{5 y}$ were obtained in lower yields due to the poor conversion of the aldehyde substrates even if higher catalyst loading was applied.

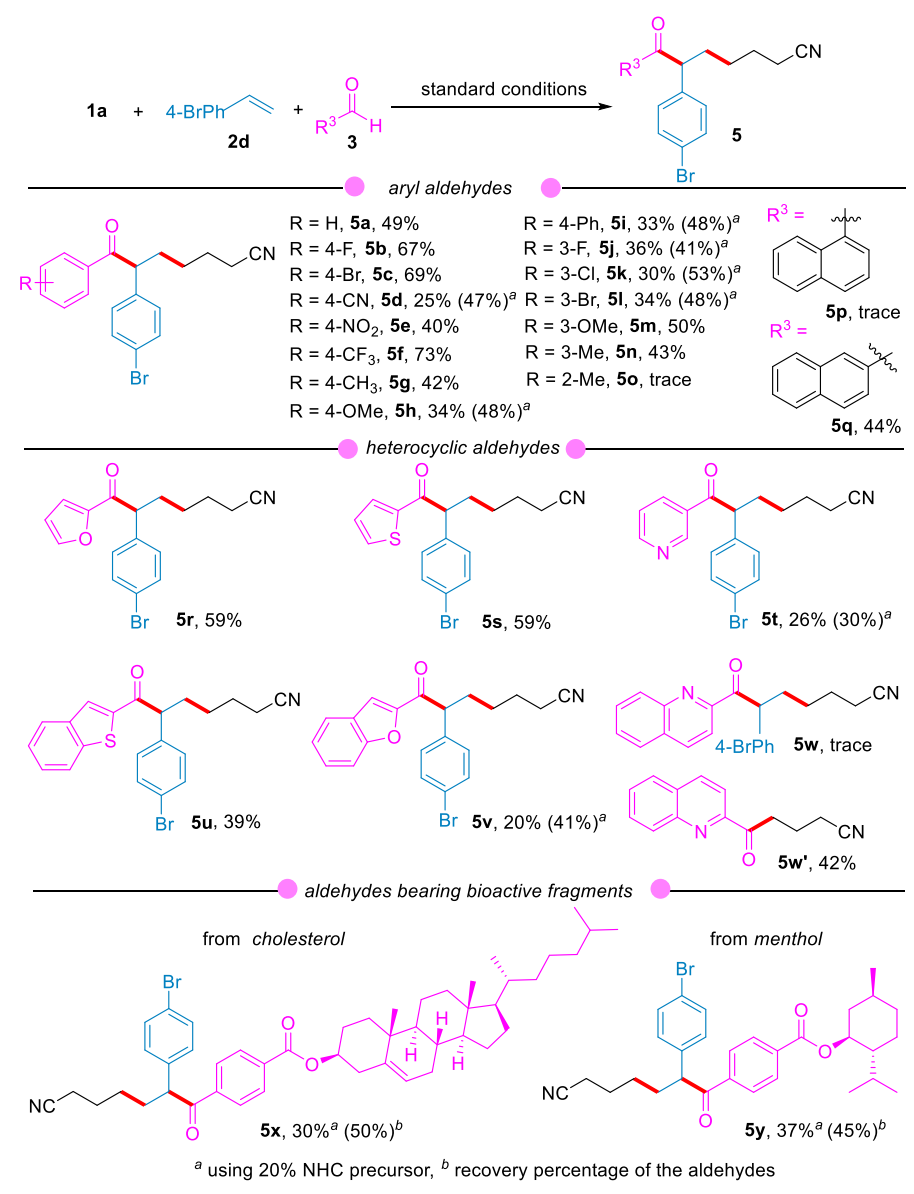

Scheme 3. Substrate scope for diverse aryl aldehydes.

Next, we turned our attention to the scope of oxime esters (Scheme 4). Cyclobutanone oxime esters bearing substituted benzyl groups at a-position (6a-6h) were well tolerated to the optimized condition, affording the corresponding aliphatic keto-nitriles in satisfactory yields but with poor diastereoselectivity. $\alpha$-Substituted cyclobutanone oxime esters always exhibited excellent regio-selectivity due to the tendency to form more stable alkyl radicals. Other than $\alpha$-substituted cyclobutanone oxime esters, several other representative $\beta$-substituted cycloketone oxime esters were also checked. To our delight, $\beta$-methoxycarbonyl substituted oxime ester, oxetan-3-one oxime ester and $\mathrm{N}$-Boc-azetidin-3-one oxime were also applicable to the optimal condition, affording products $\mathbf{6 i}, \mathbf{6} \mathbf{j}$ and $\mathbf{6 k}$ respectively, in lower to moderate yields. However, the coupling of the thietan3-one oxime ester substrate lead to a significantly decreased yield due to the complexity of the reaction (6I). It is noteworthy that the reaction of the oxime ester bearing a menthol fragment worked equally well to introduce the aliphatic chain with four functional groups in a single step $(6 \mathrm{~m})$. 


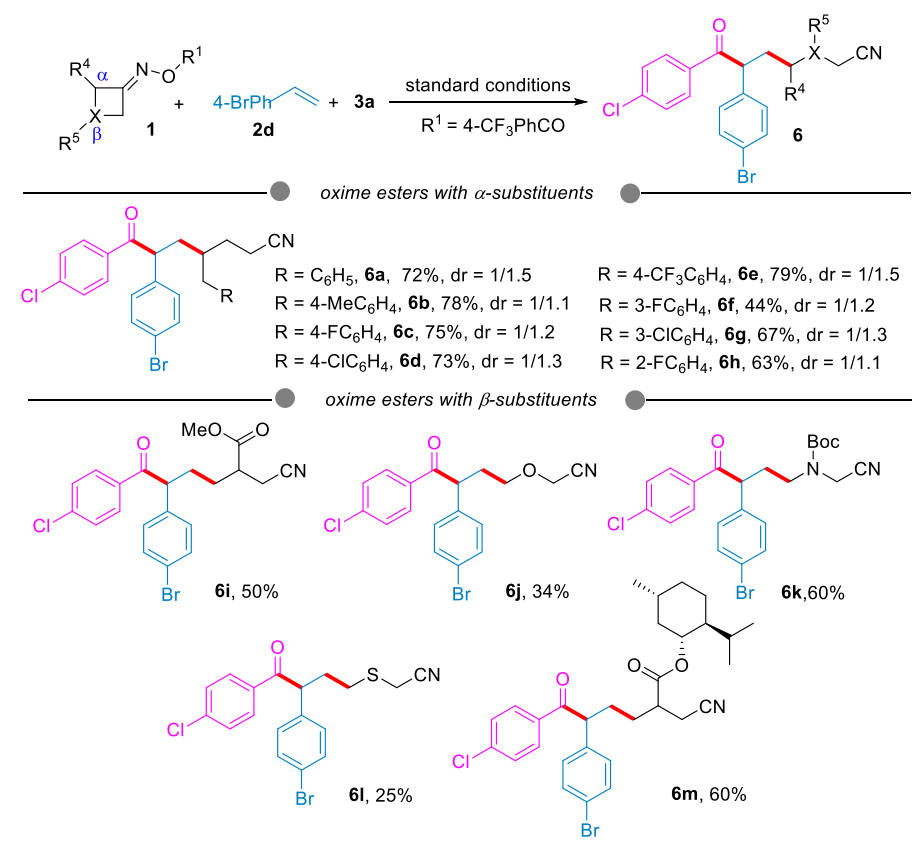

Scheme 4. Substrate scope for cycloketone oxime esters.

Literature survey indicated that the keto-alkylated nitriles represented a class of important synthetic intermediates ${ }^{16}$, thus, investigation with the scale-up reaction and the downstream applications using product 4a as the starting material were then conducted (Scheme 5). The reaction with $3 \mathrm{mmol}$ scale under the standard conditions gave rise to product $\mathbf{4 a}$ in a slightly decreased yield (60\%). Reduction of $\mathbf{4 a}$ with $\mathrm{NaBH}_{4}$ afforded alcohol $7 \mathbf{a}$ in $95 \%$ yield. $\quad 4 a$ could be also smoothly hydrolyzed to carboxylic acid $\mathbf{7 b}$ in a moderate yield, which had the potential for late-stage assembling medicinal molecules, such as the PROTACs and dual-target inhibitors. In this case, late-stage decoration of $7 \mathbf{b}$ with $\mathrm{NH}_{2} \mathrm{OH}$ using a peptide coupling reagent (PCR) could lead to a HADC inhibitor skeleton $\mathbf{7 c} \cdot{ }^{17}$ Alternatively, the amidation of acid $\mathbf{7 b}$ with phenethylamine or amphetamine afforded potential PROTACs frameworks $\mathbf{7 d}$ and $7 \mathbf{e}$ in high yields. ${ }^{18}$ The above developed methods demonstrated a great potential for further medicinal and biochemical uses.

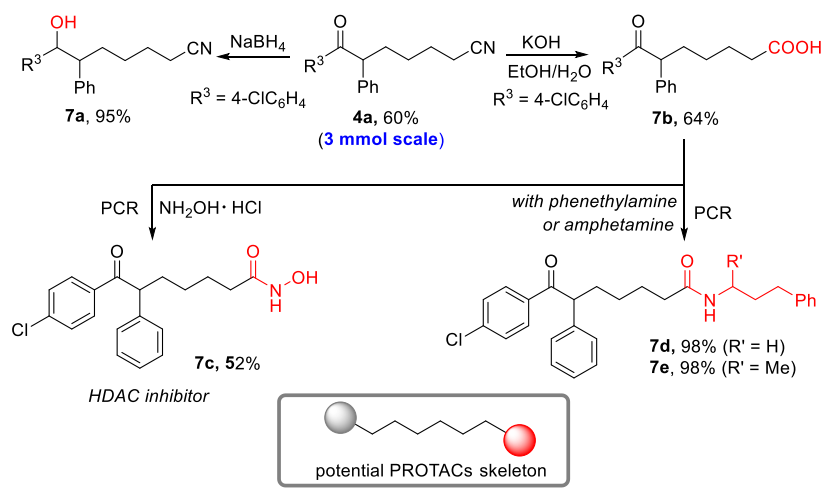

Scheme 5. Derivation of keto-alkyl nitrile $\mathbf{4 a}$ and potential application in the synthesis of the key intermediates of medicinal molecules. 
Several control experiments were then carried out to clarify the possible mechanism. First, the reaction did not work after addition of excess TEMPO under the standard conditions, which indicated a possible radical mechanism (Scheme 6a). As mentioned above, the Breslow enolate may not have enough reductive potential to reduce cyclobutanone oxime ester. It was supposed that the $\mathrm{Mg}(\mathrm{OTf})_{2}$ might have an important interaction between cyclo-oxime ester and the enolate. According to literature reports, on one hand, NHCs could reversibly bind to hard Lewis acids such as alkali metals due to the weak interaction. ${ }^{19}$ On the other hand, Lewis acids, typically like $\mathrm{Mg}^{2+}$ might activate the substrates with an $\mathrm{NHC}$ catalyst simultaneously. ${ }^{20}$ The ultraviolet absorption (UV) experiment well supported this assumption. Compared to the curves for oxime ester and Breslow intermediate individually, an extra absorption peak around $350 \mathrm{~nm}$ was observed when oxime ester and Breslow intermediate were charged together (Figure 2a, red line vs blue/green line). Meantime, blue shift was observed when the oxime ester/Breslow intermediate mixture was charged with $\mathrm{Mg}(\mathrm{OTf})_{2}$ (Figure 2b, pink line vs red line). Likewise, the cyclic voltammograms (CV) test was taken for Breslow intermediate and Breslow intermediate/Mg(OTf) $)_{2}$ mixture, respectively. The Breslow intermediate/Mg(OTf $)_{2}$ group (blue line) showed two distinctively separated quasi-reversible redox-waves (Figure 2c). Additionally, significant oxidative peak shift could be figured out by comparing the two curves with or without adding $\mathrm{Mg}(\mathrm{OTf})_{2}$ (blue line vs green line). It was also found that the peak current for Breslow intermediate group (green line) was lower than the Breslow intermediate/ $\mathrm{Mg}(\mathrm{OTf})_{2}$ group (blue line). The $\mathrm{CV}$ experiments also supported that $\mathrm{Mg}^{2+}$ had interaction with Breslow intermediate. In collection of literatures and these experimental evidences, $\mathrm{Mg}^{2+}$ might bind to both the Breslow intermediate and oxime ester, therefore making substrates redox "matched". Next, an "On-Off" experiment was conducted with intermittence heating (Figure $2 \mathrm{~d}$, heating for 20 mins, stop heating for 20 min and take a loop). As expected, the reaction proceeded faster during the heating period and slowed down without heating. The heating energy might be the driving force to accelerate the single electron transfer (SET) between the Mg/oxime ester/Breslow EDA complex (intermediate IV in Scheme 7).

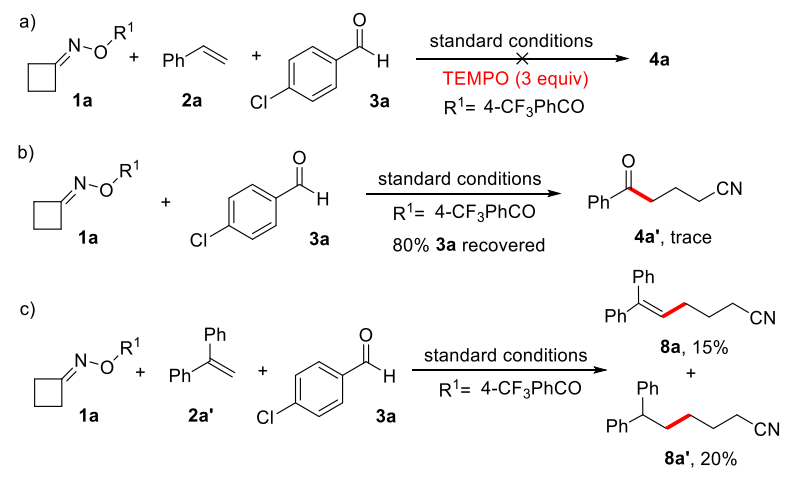

Scheme 6. Controlled experiments. 


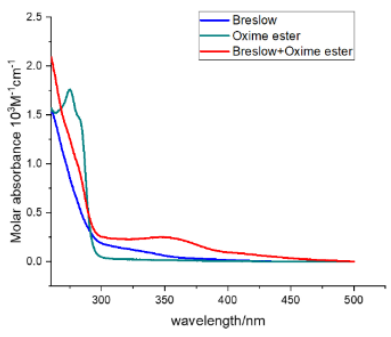

(a)

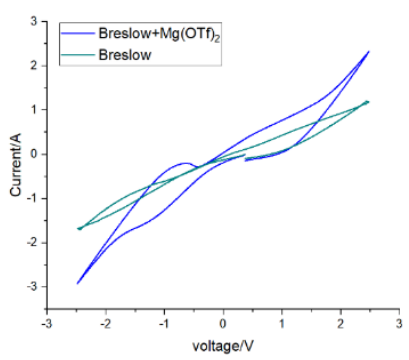

(c)

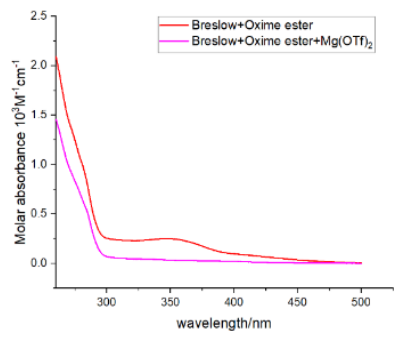

(b)

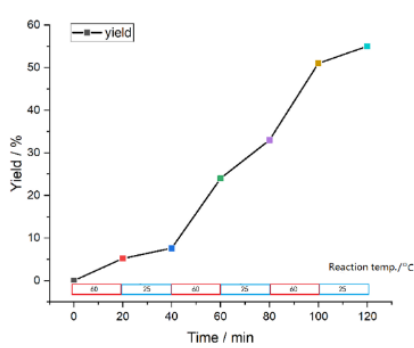

( d )

Figure 2. (a) ultraviolet absorption curve, blue line: Breslow intermediate (aldehyde, NHC precursor and base, pre-mixed in DMAc); green line: oxime ester (cyclobutanone oxime ester in DMAc); red line: mixture of oxime ester and Breslow intermediate in DMAc. (b) ultraviolet absorption curve, red line: mixture of oxime ester and Breslow intermediate in DMAc, pink line: mixture of oxime ester and Breslow intermediate and $\mathrm{Mg}(\mathrm{OTf})_{2}$ in DMAc. (c) cyclic voltammograms, blue line: Breslow intermediate; green line: mixture of Breslow intermediate and $\mathrm{Mg}(\mathrm{OTf})_{2}$. (d) "on-off" experiment: tracking of the yields for different reaction time, heating and without heating every 20 mins. All the yields were recorded based on ${ }^{1} \mathrm{H}$ NMR using mesitylene as the internal standard.

However, it was found that in the absence of styrene $\mathbf{2 a}$, the two-component reaction between substrate $\mathbf{1 a}$ and 3a did not perform well (Scheme 6b), and only trace amount of product 4a' was observed with a large quantity of recovered aldehyde $\mathbf{3 a}$. Additionally, the desired three-component product was not observed when replacing styrene $\mathbf{2 a}$ with ethene-1,1-diyldibenzene $\mathbf{2 a}$ '. Instead, a mixture of two-component coupling products $\mathbf{8 a}$ and $\mathbf{8} \mathbf{a}$ ' was obtained (Scheme $6 \mathrm{c}$ ). Therefore, the role of styrene in this reaction system was supposed to capture the nitrile alkyl radical generated after the above-mentioned SET process to form the more stable benzyl radical (intermediate VII in Scheme 7). However, as for 2a', the more sterically hindered tertiary benzyl radical generated upon the combination of the nitrile alkyl radical with 2a' may block the further interaction with Breslow enolate radical (intermediate $\mathbf{V}$ in Scheme 7). So, this "matched" radical reactivity might be another important factor for the success of this reaction.

Finally, a proposed radical-radical crossover mechanism was illustrated based on experimental results and literatures in scheme $7 .{ }^{[13,21]}$ Initially, the deprotonated Breslow intermediate III was formed through the combination of the aldehyde and NHC-I under basic condition. The coordination of $\mathrm{Mg}^{2+}$ with cyclobutanone oxime ester and intermediate III produced a sandwich type complex IV. Next, the thermal controlled single electron transfer event between the enolate and cyclobutanone oxime ester provided an NHC-bound radical V 
and a nitrile alkyl radical $\mathbf{V I}$, respectively.22 The nitrile alkyl radical was quickly captured by an olefin, forming a more stable benzyl radical VII. Subsequent radical-radical coupling between VII and V afforded intermediate VIII, which was further transformed to the final product with the regeneration of an NHC for the next catalytic cycle.

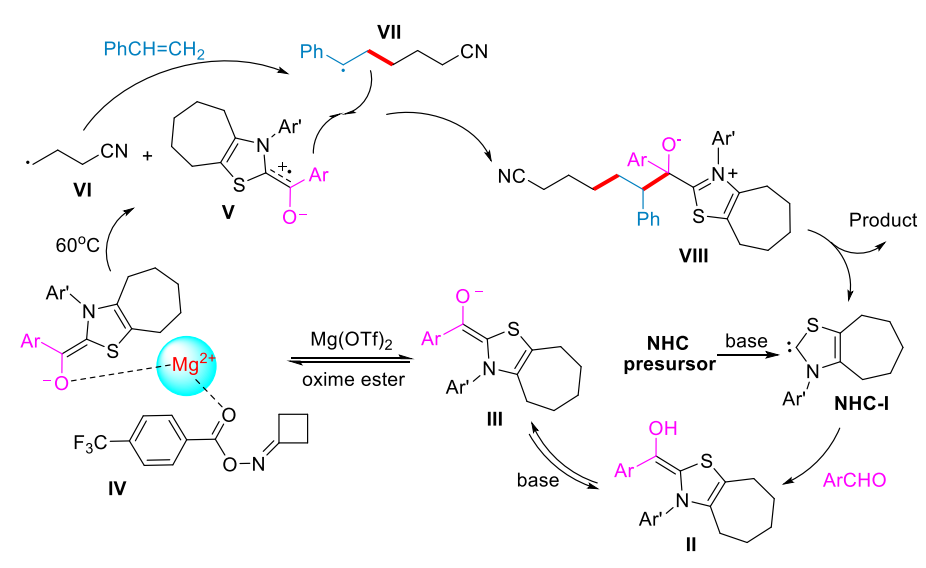

Scheme 7 Proposed mechanism

In summary, we have described an $\mathrm{NHC/Mg} \mathrm{co-catalyzed} \mathrm{olefin} \mathrm{alkylacylation} \mathrm{protocol} \mathrm{to} \mathrm{access} \mathrm{structurally}$ diverse multi-functionalized aliphatic nitrile molecules. The proposed Mg/cyclo-oxime ester/Breslow intermediate EDA complex might account for the observed reactivity. Moreover, given the simple reaction manipulation, free of transition metal catalyst, free of photo-redox catalyst and wide substrates generality, the developed protocol might have widespread use in the field of molecule assembly, PROTACs and dual target inhibitor library synthesis.

\section{Acknowledgements}

We warmly thank National Natural Science Foundation of China (21572270, 21702232), and the "Double FirstClass" Uni-versity Project (CPU2018GY02 and CPU2018GY35) for financial support of this work.

[1] T. Liu, Y. Wan, Y. Xiao, C. Xia, G. Duan, J. Med. Chem. 2020, 63, 8977-9002 and references therein.

[2] a) M. U. Brianna, M. K. Andrea, ACS Sus. Chem. Eng. 2018, 6, 3659-3668; b) F. Stempfle, P. Ortmann, S. Mecking, Chem. Rev. 2016, 116, 4597-4641.

[3] A. Krzykawska, M. Szwed, J. Ossowski, P. Cyganik, J. Phys. Chem. C, 2018, 122, 919-928.

[4] For selected recent reviews, see: a) S. S. Bhojgude, A. Bhunia, A. T. Biju, Acc. Chem. Res. 2016, 49, 1658-1670; b) C. G. Neochoritis, T. Zhao, A. Dömling, Chem. Rev. 2019, 119, 1970-2042; c) S. Javanbakht, A. Shaabani, ACS Appl. Bio Mater. 2020, 3, 156-174; d) L. Reguera, D. G. Rivera, Chem. Rev. 2019, 119, 9836-9860.

[5] For selected reviews on dicarbofunctionalization of alkenes, see: a) J. Lin, R. -J. Song, M. Hu, J. H. Li, Chem. Rec. 2019, 19, 440-451; b) Y. Li, D. Wu, H. Cheng, G. Yin, Angew. Chem., Int. Ed. 2020, 59, 7990-8003; c) S. KC, R. Giri, J. Org. Chem. 2018, 83, 3013-3022; d) J. Derosa, O. Apolinar, T. Kang, V. Tran, K. Engle, Chem. Sci. 2020, 11, 4287-4296; e) H.-Y. Tu, S. Zhu, F.L. Qing, L. Chu, Synthesis 2020, 52, 1346-1356.

[6] For selected metal catalyzed $\mathrm{C}\left(\mathrm{sp}^{3}\right)-\mathrm{C}$ dicarbofunctionalization of alkenes beyond Pd, Ni, see: a) F. J. R. Klauck, H. Yoon, M. J. James, M. Lautens, F. Glorius, ACS Catal. 2019, 9, 236-241; b) X. Yong, Y.-F. Han, Y. Li, R.-J. Song, J.-H. Li, Chem. Commun. 2018, 54, 12816-12819; c) X.-L. Lv, C. Wang, Q.-L. Wang, W. Shu, Org. Lett. 2019, 21, 56-59.

[7] For selected Pd-catalyzed $\mathrm{C}\left(\mathrm{sp}^{3}\right)-\mathrm{C}$ dicarbofunctionalization of alkenes, see: a) G. Yin, X. Mu, G. Liu, Acc. Chem. Res. 2016, 49, 2413-2423; b) Z.-M. Zhang, B. Xu, L. Wu, Y. Wu, Y. Qian, L. Zhou, Y. Liu, J. Zhang, Angew. Chem., Int. Ed. 2019, 58, $14653-14659$. 
[8] For recent nickel-catalyzed $\mathrm{C}\left(\mathrm{sp}^{3}\right)-\mathrm{C}$ dicarbofunctionalization of alkenes, see: a) A. García-Domínguez, R. Mondal, C. Nevado, Angew. Chem., Int. Ed. 2019, 58, 12286-12290; b) R. S. Mega, V. K. Duong, A. Noble, V. K. Aggarwal, Angew. Chem., Int. Ed. 2020, 59, 4375-4379; c) M. W. Campbell, J. S. Compton, C. B. Kelly, G. A. Molander, J. Am. Chem. Soc. 2019, 141, 20069-20078; d) T. Ma, Y. Chen, Y. Li, Y. Ping, W. Kong, ACS Catal. 2019, 9, 9127-9133; e) Y. Jin, C. Wang, Angew. Chem., Int. Ed. 2019, 58, 6722-6726.

[9] For selected reviews, see: a) X.-Y. Chen, Z.-H. Gao, S. Ye, Acc. Chem. Res. 2020, 53, 690-702; b) D. M. Flanigan, F. RomanovMichailidis, N. A. White, T. Rovis, Chem. Rev. 2015, 115, 9307-9387; c) M. N. Hopkinson, C. Richter, M. Schedler, F. Glorius, An overview of $\mathrm{N}$-heterocyclic carbenes. Nature 2014, 510, 485-496; d) C. Zhang, J. F. Hooper, D. W. Lupton, ACS Catal. 2017, 7, 2583-2596; e) S. Mondal, S. R. Yetra, S. Mukherjee, A. T. Biju, Acc. Chem. Res. 2019, 52, 425-436.

[10] For reviews on radical NHC catalysis, see: a) T. Ishii, K. Nagao, H. Ohmiya, Chem. Sci. 2020. 11, 5630-5636; b) H. Ohmiya, ACS Catal. 2020, 10, 6862-6869; c) Q. Liu, X.-Y. Chen, Org. Chem. Front., 2020, 7, 2082-2087; d) J. Liu, X.-N. Xing, J.-H. Huang, L.-Q. Lu, W.-J. Xiao, Chem. Sci. 2020, DOI:10.1039/D0SC03595E.

[11] a) T. Ishii, K. Ota, K. Nagao, H. Ohmiya, J. Am. Chem. Soc. 2019, 141, 14073-14077; b) K. Ota, K. Nagao, H. Ohmiya, Org. Lett. 2020, 22, 3922-3925; c) I. Kim, H. Im, H. Lee, S. Hong, Chem. Sci. 2020, 11, 3192-3197; d) J.-L. Li, Y.-Q. Liu, W.-L. Zou, R. Zeng, X. Zhang, Y. Liu, B. Han, Y. He, H.-J. Leng, Q.-Z. Li, Angew. Chem., Int. Ed., 2020, 59, 1863-1870; e) B. Zhang, Q. Peng, D. Guo, J. Wang, Org. Lett., 2020, 22, 443-447; f) H.-B. Yang, Z.-H. Wang, J.-M. Li, C. Wu, Chem. Commun., 2020, 56, 38013804.

[12] For selected references on oxime esters, see: a) J. Lou, J. Ma, B. H. Xu, Y. G. Zhou, Z. Yu, Org. Lett. 2020, 22, 5202-5206; b) D. Yang, H. Huang, M. H. Li, X. J. Si, H. Zhang, J. L. Niu, M. P. Song, Org. Lett. 2020, 22, 4333-4338; c) X. Y. Yu, J. Chen, H. W. Chen, W. J. Xiao, J. R. Chen, Org. Lett. 2020, 22, 2333-2338; d) X. Y. Yu, Q. Q. Zhao, J. Chen; W. J. Xiao, J. R. Chen, Acc. Chem. Res. 2020, 53, 1066-1083; e) B. Zhao, Y. Wu, Y. Yuan, Z. Shi, Chem. Commun. 2020, 56, 4676-4679; f) Y. Deng, C. Zhao, Y. Zhou, H. Wang, X. Li, G.-J. Cheng, J. Fu, Org. Lett. 2020, 22, 3524-3530.

[13] T. Ishii, Y. Kakeno, K. Nagao, H. Ohmiya, J. Am. Chem.Soc. 2019, 141, 3854-3858.

[14] X. Zhao, M. Tian, L. Ji, J. Liu, K. Lu, Org. Lett. 2020, 22, 863-866.

[15] W. Zhang, Chem. Rev. 2009, 109, 749-795.

[16] K. B. Charles, S. K. Richard. J. Am. Chem. Soc. 1950, 72, 277-27917.

[17] T. C. S. Ho, A. H. Y. Chan, A. Ganesan, J. Med. Chem. 2020, DOI:10.1021/acs.jmedchem.0c00830.

[18] X. Sun, Y. Rao, Biochem. 2020, 59, 240-249.

[19] K. Nagao, H. Ohmiya, Top. Curr. Chem. 2019, 377, 35-50.

[20] D.E.A. Raup, B. Cardinal-David, D. Holte, K. A. Scheidt, Nat. Chem. 2010, 2, 766-771.

[21] G. E. M. Crisenza, D. Mazzarella, P. Melchiorre, J. Am. Chem. Soc. 2020, 142, 5461-5476.

[22] V. Regnier, E. A. Romero, F. Molton, R. Jazzar, G. Bertrand, D. Martin, J. Am. Chem. Soc. 2019, 141, 1109-1113. 